\title{
Dual nature of Ricci scalar and creation of spinless particles
}

\author{
K P SINHA and S K SRIVASTAVA* \\ Department of Physics, Indian Institute of Science, Bangalore 560 012, India \\ * Permanent address: Department of Mathematics, North Eastern Hill University Permanent \\ Campus, Umshing, Shillong 793 022, India \\ * Present address: Centre for Theoretical Studies, Indian Institute of Science, Bangalore \\ 560012 , India (short term visitor)
}

MS received 30 September 1994; revised 13 January 1995

\begin{abstract}
Manifestation of Ricci scalar like a matter field as well as a geometrical field, at high energy, has been noted earlier [9]. Here, its interaction with another scalar field is considered in four-dimensional curved space-time. This interaction leads to the production of a large number of pairs of spinless particle-antiparticle due to expansion of the early universe in the vacuum state (provided by temperature dependent Coleman-Weinberg like potential for Ricci field), where spontaneous symmetry breaking takes place.
\end{abstract}

Keywords. $\quad R^{2}$ gravity; dual nature of Ricci scalar; early Universe; particle production.

\section{PACS No. $98 \cdot 80$}

\section{Introduction}

In spite of its success at low energy (long distance), Einstein's theory of gravity is problematic at high energy. According to Hawking-Penrose theorem [1], the field equations of Einstein's theory exhibit point-like singularities, where physical laws collapse. This is the first problem. The second problem is the failure of attempts to renormalize Einstein's theory of gravity. Hence this theory needs modification at least at high energy levels. In this context $R^{2}$-gravity [2]-[10] is very interesting which incorporates the general principle of covariance and reduces to Einsteinian gravity at low energy. $R^{2}$-gravity is obtained by adding $R^{2}$-terms (like $R^{2}, R_{\mu \nu} R^{\mu \nu}$ and $R_{\mu \nu \rho \sigma} R^{\mu \nu \rho \sigma}$,where $R$ is Ricci scalar, $R_{\mu \nu}$ are components of Ricci tensor and $R_{\mu \nu \rho \sigma}$ are components of Riemann-Curvature tensor) to the Einstein-Hilbert lagrangian which is linear in $R$. According to the definition of $R, R_{\mu \nu}$ and $R_{\mu v \rho \sigma}$, one knows that these geometrical quantities involve second order derivatives of components of the metric tensor as well as square of the first order derivatives of the same with respect to space-time coordinates. Components of the metric tensor are defined as

$$
\mathrm{d} s^{2}=g_{\mu \nu} \mathrm{d} x^{\mu} \mathrm{d} x^{\nu}
$$

where $\mu, v=0,1,2,3$. It is obvious from (1.1) that $g_{\mu \nu}$ are dimensionless. So, in natural units ( $\hbar=c=k_{B}=1$, where $\hbar, c$ and $k_{B}$ have their usual meaning), the dimension of $R$, $R_{\mu \nu}$ and $R_{\mu \nu \rho \sigma}$ is (mass) ${ }^{2}$. Hence, $R^{2}$-terms are insignificant at low energy but are very important at high energy level. Importance of $R^{2}$-gravity was realized in the context of renormalizability of gravity and $R^{2}$-terms also appear as stringy correction to Einstein-Hilbert lagrangian [11]. Some authors [12] have considered lagrangian 


\section{$K P$ Sinha and $S K$ Srivastava}

containing polynomial of $R$ of order higher than two also and have obtained some interesting results. But in these theories, either coupling constants are not dimensionless or dimensionality of space-time is taken to be more than 4 . In such cases, the theory is not renormalizable. The present paper deals with $R^{2}$-gravity with action given as

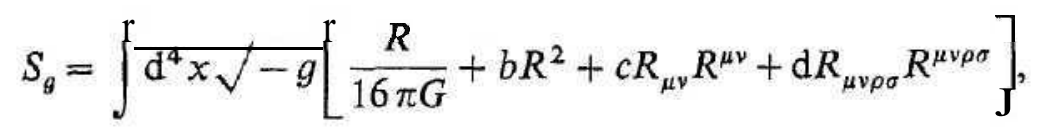

where $G$ is the gravitational constant of dimension (mass) ${ }^{-2}, g$ is the determinant of $g_{r}$ and coupling constants $(b, c$ and $d$ ) are dimensionless.

Taking the above action for gravity $\left(S_{g}\right.$, given by $\left.(1.2)\right)$, it has been discussed earlier $[9,10]$ that, at high energy, $R$ manifests itself not only as a geometrical field, but also as a spinless matter field. This kind of dual nature of $R$, at high energy, is very interesting as well as useful. In quantum field theory, fields describe elementary particles. So, Ricci scalar $R$ is supposed to describe $R$-quanta, called as Riccions [10], which are spinless bosons.

In the present paper, taking the material aspect of the Ricci scalar, the main focus lies on interactions of spinless Bose field $\tilde{R}=\eta R$ (where $\eta$ is a parameter of unit magnitude and length dimension) with another scalar field 0 and its implications in the inflationary model obtained after spontaneous symmetry breaking in the theory with temperature dependent Coleman-Weinberg like potential for $\tilde{R}$. The interaction term is defined as $L_{I}=\frac{1}{2} \lambda \widetilde{R}^{2} \phi^{2}$. When $\widetilde{R}$ undergoes spontaneous symmetry breaking it leads to a phase transition from the state $\langle R\rangle=0$ to $\langle\tilde{R}\rangle= \pm a$ ( $a$ is the spontaneous symmetry breaking mass scale and $\langle\widetilde{R}\rangle$ is the vacuum expectation value of $\tilde{R}$ which is homogeneous). So long as temperature $T$ is above or equal to the critical temperature $T_{c},\langle\tilde{R}\rangle$ remains confined to the state $\langle\tilde{R}\rangle=0$. But as $T$ falls very much below $T_{c},\langle\tilde{R}\rangle$ tunnels through the temperature barrier $\left(T=T_{c}\right)$ and acquires a nonzero constant value $\langle\tilde{R}\rangle=+a$. As a result, other scalar field $\phi$ is massless in the state $\langle\widetilde{R}\rangle=0$ and acquires non-vanishing mass in the state $\langle\widetilde{R}\rangle= \pm \sigma$. It is demonstrated that a large number of particle-antiparticle pairs are created due to exponentially expanding model of the early universe in the state $\langle\tilde{R}\rangle=+a$.

The paper is organized as follows. Section 2 deals with spontaneous symmetry breaking using temperature dependent Coleman-Weinberg-like potential for $\tilde{R}$ in place of temperature dependent Higgs-like potential used in [9]. Taking the geometrical aspect of $\widetilde{R}$, it is found that the state $\langle R \widetilde{R}=-G$ behaves like bumps in the rapidly expanding model of the early universe given by the state $\langle\bar{R}\rangle=+a$. In this section, it is also discussed that a huge amount of energy will be released as a result of spontaneous symmetry breaking. In $\S 3$, the Klein-Gordon equation for $\phi$ is derived and normalized solutions of the same have been obtained. Section 4 contains discussions concerning creation of particles. Section 5 is the concluding section where energy of the created particles is calculated. The natural units are used throughout the paper.

\section{Spontaneous symmetry breaking}

The four-dimensional generalization of Gauss-Bonnet theorem implies that

$$
\int \mathrm{d}^{4} x \sqrt{-g}\left(R_{\mu \nu \rho \sigma} R^{\mu \nu \rho \sigma}-4 R_{\mu v} R^{\mu \nu}+R^{2}\right)=x,
$$




\section{$K P$ Sinha and $S K$ Srivastava}

containing polynomial of $R$ of order higher than two also and have obtained some interesting results. But in these theories, either coupling constants are not dimensionless or dimensionality of space-time is taken to be more than 4 . In such cases, the theory is not renormalizable. The present paper deals with $R^{2}$-gravity with action given as

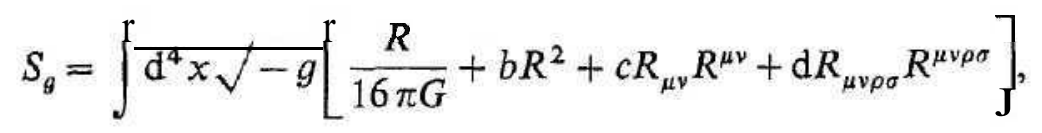

where $G$ is the gravitational constant of dimension (mass) ${ }^{-2}, g$ is the determinant of $g_{r}$ and coupling constants $(b, c$ and $d$ ) are dimensionless.

Taking the above action for gravity $\left(S_{g}\right.$, given by $\left.(1.2)\right)$, it has been discussed earlier $[9,10]$ that, at high energy, $R$ manifests itself not only as a geometrical field, but also as a spinless matter field. This kind of dual nature of $R$, at high energy, is very interesting as well as useful. In quantum field theory, fields describe elementary particles. So, Ricci scalar $R$ is supposed to describe $R$-quanta, called as Riccions [10], which are spinless bosons.

In the present paper, taking the material aspect of the Ricci scalar, the main focus lies on interactions of spinless Bose field $\tilde{R}=\eta R$ (where $\eta$ is a parameter of unit magnitude and length dimension) with another scalar field 0 and its implications in the inflationary model obtained after spontaneous symmetry breaking in the theory with temperature dependent Coleman-Weinberg like potential for $\tilde{R}$. The interaction term is defined as $L_{I}=\frac{1}{2} \lambda \widetilde{R}^{2} \phi^{2}$. When $\widetilde{R}$ undergoes spontaneous symmetry breaking it leads to a phase transition from the state $\langle R\rangle=0$ to $\langle\tilde{R}\rangle= \pm a$ ( $a$ is the spontaneous symmetry breaking mass scale and $\langle\widetilde{R}\rangle$ is the vacuum expectation value of $\tilde{R}$ which is homogeneous). So long as temperature $T$ is above or equal to the critical temperature $T_{c},\langle\tilde{R}\rangle$ remains confined to the state $\langle\tilde{R}\rangle=0$. But as $T$ falls very much below $T_{c},\langle\tilde{R}\rangle$ tunnels through the temperature barrier $\left(T=T_{c}\right)$ and acquires a nonzero constant value $\langle\tilde{R}\rangle=+a$. As a result, other scalar field $\phi$ is massless in the state $\langle\widetilde{R}\rangle=0$ and acquires non-vanishing mass in the state $\langle\widetilde{R}\rangle= \pm \sigma$. It is demonstrated that a large number of particle-antiparticle pairs are created due to exponentially expanding model of the early universe in the state $\langle\tilde{R}\rangle=+a$.

The paper is organized as follows. Section 2 deals with spontaneous symmetry breaking using temperature dependent Coleman-Weinberg-like potential for $\tilde{R}$ in place of temperature dependent Higgs-like potential used in [9]. Taking the geometrical aspect of $\widetilde{R}$, it is found that the state $\langle R \widetilde{R}=-G$ behaves like bumps in the rapidly expanding model of the early universe given by the state $\langle\bar{R}\rangle=+a$. In this section, it is also discussed that a huge amount of energy will be released as a result of spontaneous symmetry breaking. In $\S 3$, the Klein-Gordon equation for $\phi$ is derived and normalized solutions of the same have been obtained. Section 4 contains discussions concerning creation of particles. Section 5 is the concluding section where energy of the created particles is calculated. The natural units are used throughout the paper.

\section{Spontaneous symmetry breaking}

The four-dimensional generalization of Gauss-Bonnet theorem implies that

$$
\int \mathrm{d}^{4} x \sqrt{-g}\left(R_{\mu \nu \rho \sigma} R^{\mu \nu \rho \sigma}-4 R_{\mu v} R^{\mu \nu}+R^{2}\right)=x,
$$




\section{$K P$ Sinha and $S K$ Srivastava}

The vacuum states are given by

$$
\left(\frac{\partial V^{T}}{\partial \tilde{R}}\right)_{\tilde{R}=\langle\tilde{R}\rangle}=0 \text { or } \quad 4 A\langle\tilde{R}\rangle^{3} \ln \frac{\langle\tilde{R}\rangle}{\sigma^{2}}+2 C T^{2}\langle\tilde{R}\rangle=0 .
$$

Equation (2.5) yields

where

$$
\langle\tilde{R}\rangle=\left\{\begin{array}{cc}
0, & \text { when } T \geqslant T_{\mathrm{v}} \\
\pm\left[\frac{C}{2 A}\left(T_{\mathrm{c}}^{2}-T^{2}\right)\right]^{1 / 2}, & \text { when } T \leqslant T_{\mathrm{c}}
\end{array}\right.
$$

$$
T_{\mathrm{c}}^{2}=\frac{2 A}{C} \sigma^{2} \text {. }
$$

So, when $T \ll T_{\mathrm{c}}$

$$
\langle\tilde{R}\rangle= \pm a \text {. }
$$

A spatially homogeneous, flat and isotropic cosmological model of the early universe is given by the Robertson-Walker line-element

$$
\mathrm{ds}^{2}=\mathrm{d} t^{2}-a^{2}(t)\left[(\mathrm{d} x)^{2}+(\mathrm{d} y)^{2}+(\mathrm{d} z)^{2}\right] .
$$

The geometrical definition of $\tilde{R}$ in the model, given by the line-element (2.10), yields

$$
\left.\tilde{R}=6 n \mid \stackrel{\ddot{a}}{-}+\left(\frac{\dot{a}}{a}\right)^{2}\right]
$$

where dot denotes derivative with respect to cosmic time $t$. $\tilde{R}$ given by (2.11), is independent of spatial coordinates, so $R=\langle R \tilde{\rangle}$. Now, the ordinary differential equation, in the state $\langle\tilde{R}\rangle=0$, is given as

$$
\frac{a}{a}+\frac{(\dot{a})^{2}}{(a)}=\mathbf{O}
$$

which integrates to

$$
a^{2}=\tilde{\alpha}+\frac{t}{t_{\mathrm{pl}}}
$$

where $\mathrm{a}$ is an integration constant rand $t_{\mathrm{pl}}$ is the Planck time. Like ref. [9] on taking $V^{T}(\tilde{R})$ as temperature-dependent Coleman-Weinberg potential, the energy condition is not violated but $\boldsymbol{R}^{\mathbf{2}}$-terms modify Einstein field equations. For the modified situation, Hawking-Penfose theorem is not applicable. Hence singularity can be avoided. This modification seems at and above the energy scale where $R^{2}$ terms in $S_{g}$ are important.

The expansion, obeying the rule given by (2.13), is adiabatic. Hence, temperature will fall as

$$
\boldsymbol{s}=\frac{\text { constant }}{\left[\tilde{\alpha}+\left(t / t_{\mathrm{p} 1}\right)\right]^{1 / 2}}
$$

Thus, one finds that temperature falls according to (114) in the symmetric state $\langle R\rangle=0$. As phase transition is expected below Planck scale, the critical temperature $T_{\mathrm{c}}$ will be less than $T_{\text {Planck }}$ which is possible at time $t_{\mathrm{c}}>t_{\mathrm{pl}}$, according to rule given 


\section{Dual nature of Ricci scalar}

by (2.14). When temperature falls very much below $T_{\mathrm{c}}$ i.e. $T \ll T_{\mathrm{c}}\langle\tilde{R}\rangle$ acquires a nonzero constant value $a$ and spontaneous symmetry breaking takes place at time $t_{0}>t_{\mathrm{c}}$.

Again taking the geometrical definition of $\tilde{R}$ given by (2.10), one gets the ordinary differential equation

$$
\frac{\ddot{a}}{a}+\left(\frac{\dot{a}}{a}\right)^{2}=\frac{\sigma}{6 \eta}
$$

yielding the solution

$$
a^{2}=a_{0}^{2} \sinh \left[\frac{2}{\eta a_{0}^{2}}\left(t-t_{0}\right)+0.89\right]
$$

where $a_{0}^{2}=(12 / \sigma \eta)^{1 / 2}$. Thus, one finds non-adiabatic expansion in the state $\langle\widetilde{R}\rangle=a$. In the case $\langle\tilde{R}\rangle--\sigma$, the ordinary differential equation will be

$$
\frac{\ddot{a}}{a}+\left(\frac{\dot{a}}{a}\right)^{2}=-\frac{\sigma}{6 \eta}
$$

yielding the solution

$$
a^{2}-a_{0}^{2} \sin \left[\frac{2}{\eta a_{0}^{2}}\left(t-t_{0}\right)+\frac{\pi}{2}\right\rfloor
$$

which implies that $0<a(t) \leqslant a_{0}$ (as discussed above $a(t) \neq 0$ and $a(t)$, being scale factor, cannot be negative or complex number). Thus, the state $\langle R . \overline{\rangle}=-\sigma$ may behave like a small bubble of size $a(t)$ such that $r \leqslant a(t) \leqslant a_{0}$ (here $\mathrm{r}$ is a very small non-zero number). It is interesting to see (from (2.18)) that at $\mathrm{t}=t_{0}, a=a_{0}$. As $t$ increases, $a(t)$ decreases to the smallest non-zero number $r$ at $t=t_{0}+\left(\eta a_{0}^{2} \pi / 4\right)$ and it remains equal to $\mathrm{r}$ till $t=t_{0}+\left(3 \eta a_{0}^{2} \pi / 4\right)$. When $\mathrm{t}>t_{0}+\left(2 \eta a_{0}^{2} \pi / 4\right), a(t)$ increases to $a_{0}$ at time $t=t_{0}+\eta a_{0}^{2} \pi$. This cycle keeps on repeating, so long as $R^{2}$-terms are significant in gravitational dynamics. Thus it is interesting to see that the state $\langle R\rangle=-a$ behaves like bumps in the cosmological model of the early universe, given by the state $\langle\widetilde{R}\rangle=a$, which expands rapidly with scale factor $a(\mathrm{t})$ obeying $(2.16)$.

Another interesting point (which is important to mention here) is that at $t=t_{0}$, when $T \ll T_{\mathrm{c}}$, a huge amount of energy will get released with density

$$
\begin{aligned}
V^{T}(0)-V^{T}(\sigma) & =\frac{1}{2} A \sigma^{4}-C T^{2} \sigma^{2} \\
& =C \sigma^{2}\left(\frac{T_{\mathrm{c}}^{2}}{4}-\rightarrow \frac{1}{2}, A\right.
\end{aligned}
$$

The gravitational energy so released will be converted into particles and radiation increasing entropy of the universe.

\section{3. $\tilde{R}$ and $\phi$ interaction and solution of Klein-Gordon equation for $\phi$}

Manifestation of material aspect of Ricci scalar, at high energy, encourages one to study a theory involving interaction of $\widetilde{R}$ and $\phi$. The action for such a theory, in 


\section{Dual nature of Ricci scalar}

by (2.14). When temperature falls very much below $T_{\mathrm{c}}$ i.e. $T \ll T_{\mathrm{c}}\langle\tilde{R}\rangle$ acquires a nonzero constant value $a$ and spontaneous symmetry breaking takes place at time $t_{0}>t_{\mathrm{c}}$.

Again taking the geometrical definition of $\tilde{R}$ given by (2.10), one gets the ordinary differential equation

$$
\frac{\ddot{a}}{a}+\left(\frac{\dot{a}}{a}\right)^{2}=\frac{\sigma}{6 \eta}
$$

yielding the solution

$$
a^{2}=a_{0}^{2} \sinh \left[\frac{2}{\eta a_{0}^{2}}\left(t-t_{0}\right)+0.89\right]
$$

where $a_{0}^{2}=(12 / \sigma \eta)^{1 / 2}$. Thus, one finds non-adiabatic expansion in the state $\langle\widetilde{R}\rangle=a$. In the case $\langle\tilde{R}\rangle--\sigma$, the ordinary differential equation will be

$$
\frac{\ddot{a}}{a}+\left(\frac{\dot{a}}{a}\right)^{2}=-\frac{\sigma}{6 \eta}
$$

yielding the solution

$$
a^{2}-a_{0}^{2} \sin \left[\frac{2}{\eta a_{0}^{2}}\left(t-t_{0}\right)+\frac{\pi}{2}\right\rfloor
$$

which implies that $0<a(t) \leqslant a_{0}$ (as discussed above $a(t) \neq 0$ and $a(t)$, being scale factor, cannot be negative or complex number). Thus, the state $\langle R . \overline{\rangle}=-\sigma$ may behave like a small bubble of size $a(t)$ such that $r \leqslant a(t) \leqslant a_{0}$ (here $\mathrm{r}$ is a very small non-zero number). It is interesting to see (from (2.18)) that at $\mathrm{t}=t_{0}, a=a_{0}$. As $t$ increases, $a(t)$ decreases to the smallest non-zero number $r$ at $t=t_{0}+\left(\eta a_{0}^{2} \pi / 4\right)$ and it remains equal to $\mathrm{r}$ till $t=t_{0}+\left(3 \eta a_{0}^{2} \pi / 4\right)$. When $\mathrm{t}>t_{0}+\left(2 \eta a_{0}^{2} \pi / 4\right), a(t)$ increases to $a_{0}$ at time $t=t_{0}+\eta a_{0}^{2} \pi$. This cycle keeps on repeating, so long as $R^{2}$-terms are significant in gravitational dynamics. Thus it is interesting to see that the state $\langle R\rangle=-a$ behaves like bumps in the cosmological model of the early universe, given by the state $\langle\widetilde{R}\rangle=a$, which expands rapidly with scale factor $a(\mathrm{t})$ obeying $(2.16)$.

Another interesting point (which is important to mention here) is that at $t=t_{0}$, when $T \ll T_{\mathrm{c}}$, a huge amount of energy will get released with density

$$
\begin{aligned}
V^{T}(0)-V^{T}(\sigma) & =\frac{1}{2} A \sigma^{4}-C T^{2} \sigma^{2} \\
& =C \sigma^{2}\left(\frac{T_{\mathrm{c}}^{2}}{4}-\rightarrow \frac{1}{2}, A\right.
\end{aligned}
$$

The gravitational energy so released will be converted into particles and radiation increasing entropy of the universe.

\section{3. $\tilde{R}$ and $\phi$ interaction and solution of Klein-Gordon equation for $\phi$}

Manifestation of material aspect of Ricci scalar, at high energy, encourages one to study a theory involving interaction of $\widetilde{R}$ and $\phi$. The action for such a theory, in 


\section{$K P$ Sinha and $S K$ Srivastava}

curved space-time is given as

$$
\begin{aligned}
S=\int d^{4} x \sqrt{-g}\left[\frac{1}{2} g^{\mu \nu} \partial_{\mu} \tilde{R} \partial_{\nu} \tilde{R}-V^{T}(\tilde{R})\right. & +\frac{1}{2} ?^{\mu \nu} \partial_{\mu} \phi \partial_{\nu} \phi \\
& \left.-\frac{1}{2}\left(\xi \eta^{-1} \tilde{R}+\lambda \tilde{R}^{2}\right) \phi^{2}\right],
\end{aligned}
$$

where $\xi \eta{ }^{1} \tilde{R} \phi^{2}=\xi R \phi^{2}$ is the non-minimal coupling term of $\phi$ with gravity alongwithnon-minimal coupling constant $\xi$ (which is dimensionless) and $\lambda \tilde{R}^{2} \phi^{2}$ is the $\widetilde{R}-\phi$ interaction term with dimensionless coupling constant $\lambda$. Here, $\left(\xi_{\eta}{ }^{-1} \tilde{R}+\lambda \tilde{R}^{2}\right) \phi^{2}$ is the mass term which vanishes in the state $\langle R\rangle=0$ and is equal to $\left( \pm \xi \eta^{-1} \sigma+\lambda \sigma^{2}\right) \phi^{2}$ in the state $\langle\tilde{R}\rangle= \pm \sigma$. As discussed in the earlier section, the state $\langle\tilde{R}\rangle=-a$ behaves like bumps only in the exponentially expanding cosmological model, so this will not be discussed hereafter.

Imposing the condition

$$
\frac{2}{\sqrt{-g}} \frac{5 S}{\delta \phi}=0
$$

on $S$, given by (3.1), one gets the Klein-Gordon equation for $\phi$ as

$$
\left(\square+\xi \eta^{-1} \tilde{R}+\lambda \tilde{R}^{2}\right) \phi==0
$$

in the curved space-time.

For the purpose of second quantization, the general wave solution of KleinGordon equation (3.3) can be written, for discrete modes fc, as

$$
\phi=\sum_{k}\left[a_{k}^{\prime} f_{k}(t) \exp \left(-\left(i k_{a} x^{a}\right)\right)+a_{k}^{\dagger} f_{k}^{*}(t) \exp \left(+i k_{a} x^{a}\right)\right]
$$

Substituting this solution in (3.3), one gets ordinary differential equation for mode feas,

$$
\frac{1}{a^{3}(t)} \frac{\mathrm{d}}{\mathrm{d} t}\left(a^{3}(t) \frac{\mathrm{d}}{\mathrm{d} t}\right) f_{k}+\left(\frac{k^{2}}{a^{2}(t)}+\xi \eta^{-1} \tilde{R}+\lambda \tilde{R}^{2}\right) f_{k}=0
$$

in the geometry, given by the line-element (2.10). In the state $\langle\tilde{R}\rangle=\bullet \mathrm{a}, a(t)$ is given by (2.18). So, (3.5) reduces to

$$
\begin{aligned}
\ddot{f}_{k} & +\frac{3}{\eta a_{0}^{2}} \operatorname{coth}\left[\frac{2}{\eta a_{0}^{2}}\left(t-t_{0}\right)+0.89\right] f_{k} \\
& +\left[\frac{k^{2}}{a_{n}^{2} \sinh ^{2}\left\{\frac{2}{\because \cdot v}\left(t-t_{0}\right)+0.89\right\}}+\xi \eta^{-1} \sigma+\lambda \sigma^{2}\right] f_{k}=0 .
\end{aligned}
$$

Now, defining

$$
\tau=\frac{2}{\eta a_{0}^{2}}\left(t-t_{0}\right)+0.89
$$


(3.6) is written as

$$
\frac{\mathrm{d}^{2} f_{k}}{\mathrm{~d} \tau^{2}}+\frac{3}{2} \operatorname{coth} \xi \frac{\mathrm{d} f_{k}}{\mathrm{~d} \tau}+\left[\left(\frac{3 \eta^{3}}{4 \sigma}\right)^{1 / 2} \frac{k^{2}}{\sinh ^{2} \mathbf{T}}+3 \xi+3 \lambda \eta \sigma\right] f_{k}=0 .
$$

On substituting

$$
i_{k}-v_{k} \sinh { }^{\cdots} \mathrm{T}
$$

in the differential equation (3.7b), one gets the ordinary differential equation

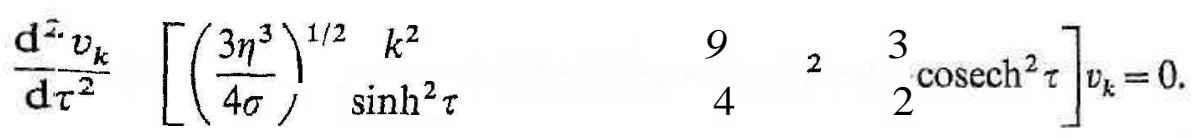

With the definition of $\tau$, given by (3.7a), one finds that $e^{-\tau} \leqslant 0 \cdot 41066$ and $e^{\tau} \geqslant 2 \cdot 4351$. So, $\sinh \mathrm{T}$ and coth $\mathrm{T}$ can be approximated as

and

$$
\sinh \tau \approx e^{\tau} / 2
$$

$$
\operatorname{coth} \tau \approx 1
$$

without any harm. As a result, the ordinary differential equation (3.9) can be written in a more convenient form

$$
\frac{d^{2} v_{\kappa}}{\mathrm{d} \tau^{2}}+\left[\left\{4\left(\frac{3 \eta^{3}}{4 \sigma}\right)^{1 / 2} k^{2}+3\right\} e^{-\iota t}+3 \xi+3 \lambda \eta \sigma-\frac{9}{16}\right] v_{k}=0 .
$$

For large $\mathrm{T}$ (3.11) can be approximated as

$$
\frac{\mathrm{d}^{2} v_{k}}{\mathrm{~d} \tau^{2}}+\left(3 \xi+3 \lambda \eta \sigma-\frac{9}{4}\right)
$$

which yields positive and negative energy solutions $e^{-i v \tau} / \sqrt{ } 2 v$ and $e^{i v \tau} / \sqrt{2 v}$ respectively, where

$$
\mathrm{v}= \pm[3 \xi+\lambda \eta \sigma-0 \cdot 19)]^{1 / 2}
$$

Equation (3.1 1) is integrated to

where

$$
v_{k}=A J_{i v}\left(K e^{-\tau}\right)+B I_{-i v}\left(K e^{-\tau}\right),
$$

$$
\begin{aligned}
& K= \pm\left[\left(12 \eta^{3} / \sigma\right)^{1 / 2} k^{2}+3\right]^{1 / 2} \\
& A=(2|v|)^{-1 / 2} 2^{i v} K^{-i v} \Gamma(1+i v), \\
& B=(2|v|)^{-1 / 2} 2^{-i v} K^{i v} \Gamma(1-i v),
\end{aligned}
$$

and $J_{l}(x)$ is Bessel's function of first kind. In (3.14), $A$ and $B$ have been evaluated using the approximation of Bessel's function for small arguments, given as,

$$
J_{l}(x) x \stackrel{\cong}{\cong} \frac{(x / 2)^{l}}{\Gamma(1+l)} .
$$




\section{$K P$ Sinha and $S K$ Srivastava}

and

$$
A J_{i v}\left(K e^{-\tau}\right) \tau \stackrel{A}{\approx} \frac{A\left(K e^{-\tau} / 2\right)^{i v}}{\Gamma(1+i v)}
$$

which should match with $e^{i v \tau} / \sqrt{2 v}$. On this kind of matching, one gets $A$, as given by (3.14c). Similarly, $B$ is also evaluated. But the solution given by (3.14) is not normalized, which is very much important to have a physically reasonable solution with correct dimensions. For the purpose of normalization, the following conditions are used

$$
\left(v_{1}, v_{1}\right)=-\left(v_{2}, v_{2}\right)=1 \text { and }\left(v_{1}, v_{2}\right)=0
$$

where $v_{1}=A J_{i v}\left(K e^{-\tau}\right)$ and $v_{2}=B J_{-i v}\left(K e^{-\tau}\right)$. The orthonormal product of two functions $\psi_{1}$ and $\psi_{2}$ is defined as

$$
\left(\psi_{1}, \psi_{2}\right)=-i \int_{t=\text { constant }} \mathrm{d}^{3} x a^{3}(t)\left[\psi_{1}(x, t) \partial_{t} \psi_{2}^{*}(x, t)-\left(\partial_{t} \psi_{1}(x, t)\right) \psi_{2}^{*}(x, t)\right] .
$$

After normalization at $t=t_{0}$, one gets

$$
v_{k}=a_{n}^{-3 / 2} V^{-1 / 2}(3 \eta / \sigma)^{1 / 4}\left[A J_{i v}\left(K e^{-\dagger} B J_{-i v}\left(K e^{-\tau}\right)\right]\right.
$$

where $V$ is the volume of 3-dimensional space. Using (3.8) and (3.18), one gets

$$
f_{k}(t)=a_{0}^{-3 / 2} V^{-1 / 2}(3 \eta / \sigma)^{1 / 4} \sinh ^{-3 / 4} \tau\left[A J_{i v}\left(K e^{-\tau}\right)+B J_{-i v}\left(K e^{-\tau}\right)\right]
$$

where $A, B$ and $K$ are given by (3.14).

\section{Production of scalar particles}

To study the spectrum of created scalar particles, in-vacuum state $\left(t \ll t_{0}\right)$ denoted as $|0\rangle_{\text {in }}$ is defined as

$$
a_{k}^{\text {in }}|0\rangle_{\text {in }}=0 \text { and }_{\text {in }}\langle 0 \mid 0\rangle_{\text {in }}=1 .
$$

The out-vacuum state $\left(t \gg t_{0}\right)$, denoted as $|0\rangle_{\text {out }}$ is defined as

$$
a_{k}^{\text {out }}|0\rangle_{\text {out }} \text { and }{ }_{\text {out }}\langle 0 \mid 0\rangle_{\text {out }}=1 \text {. }
$$

The scalar field $\phi(x, t)$ can be expanded in terms of a complete set of orthonormal functions $\phi_{k}^{\text {in }}(x, t)$ as

$$
\phi=\sum_{k}\left[a_{k}^{\mathrm{in}} \phi_{k}^{\mathrm{in}}(x, t)+a_{k}^{\mathrm{in} \dagger} \phi_{k}^{\mathrm{in} *}(x, t)\right]
$$

where $\phi_{k}^{\mathrm{in}}(x, t)=f_{k}^{\mathrm{in}}(t) \exp \left(-i k_{a} x^{a}\right)$ with its complex conjugate $\phi_{k}^{\mathrm{in} *}$ and $a_{k}^{\mathrm{in}}\left(a_{k}^{\mathrm{in}}\right)$ are annihilation (creation) operators in the in-state $|0\rangle_{\text {in }}$. $\phi$ can also be expanded, in the second complete set of orthonormal functions $\phi_{\dot{k}}^{\text {out }}(x, t)$ as

$$
\phi=\underset{k}{\mathrm{E}}\left[a_{k}^{\text {out }} \phi_{k}^{\text {out }}(x, t)+a_{k}^{\text {out }} \phi_{k}^{\text {out } *}(x, 03\right.
$$




\section{Dual nature of Ricci scalar}

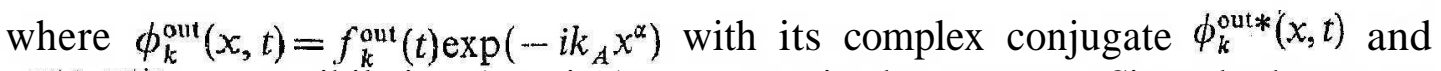
$a_{k}^{\text {out }}\left(a_{k}^{\text {out }}\right)$ are annihilation (creation) operators in the out-state. Since, both sets are complete, $\phi_{k}^{\text {out }}$ can be expanded in terms of $\phi_{k}^{\text {in }}$ and $\phi_{k}^{\text {in* }}$ as [15]-[17]

$$
\phi_{k}^{\text {out }}=\alpha_{k} \phi_{k}^{\text {in }}+\beta_{k} \phi_{k}^{\text {in* }} .
$$

The relations, given by (4.5), are known as Bogoliubov transformations. Here, $\alpha_{k}$ and $\beta_{k}$ are Bogoliubov coefficients, given as

and

$$
\alpha_{\mathrm{i}}=\left(\phi_{k}^{\mathrm{out}}, \phi_{k}^{\mathrm{in}}\right)=-i \sum_{\mathrm{J}(=£ 0} \mathrm{d}^{3} x a^{3}(t)\left[\phi_{k}^{\text {out }} \partial_{0} \phi_{k}^{\mathrm{in} *}-\left(\partial_{0} \phi_{k}^{\text {out }}\right) \phi_{k}^{\mathrm{in} *}\right]
$$

$$
\beta_{k}=\left(\phi_{k}^{\text {out }}, \phi_{k}^{\text {in } *}\right)=i \int_{J_{t=t 0}} a^{3} x a^{3}(t)\left[\phi_{k}^{\text {out }} \partial_{0} \phi_{k}^{\text {in }}-\left(\partial_{0} \phi_{k}^{\text {out }}\right) \phi_{k}^{\text {in }}\right]
$$

The Bogoliubov coefficients $\alpha_{k}$ and $\beta_{k}$ obey the condition

$$
\left|\alpha_{k}\right|^{2}-\left|\beta_{k}\right|^{2}=1
$$

The set of solutions (as $t \ll t_{0}$ ) are

$$
\begin{gathered}
\phi_{k}^{\text {in }}(x, t)=a_{0}^{-3 / 2} A V^{-1 / 2}\left[-\frac{3 \eta}{\sigma}\right]^{1 / 4} e^{+3 \tau / 4} J_{i v}\left(K e^{-\tau}\right) e^{-i k_{a} x^{\alpha}} \\
\phi_{k}^{\text {in } *}(x, t)=a_{0}^{-3 / 2} B V^{-1 / 2}\left[-\frac{3 \eta}{\sigma}\right]^{1 / 4}-J_{-i v}\left(K e^{-\tau}\right) e^{-i k_{a} x^{\alpha}} .
\end{gathered}
$$

The set of solutions (as $t \gg t_{0}$ ) are

$$
\begin{aligned}
\phi_{k}^{\text {out }}(x, t) & =a_{0}^{-3 / 2} A V^{-1 / 2}(3 \eta / \sigma)^{1 / 4} e^{-3 z / 4} J_{i v}\left(K e^{-\tau}\right) e^{-i k_{a} x^{\alpha}} \\
\phi_{k}^{\text {out } *}(x, t) & =a_{0}^{-3 / 2} B V^{-1 / 2}(3 \eta / \sigma)^{1 / 4} e^{3 \tau / 4} J_{-i v}\left(K e^{-\tau}\right) e^{i k_{a} x^{\alpha}}
\end{aligned}
$$

Now, for discussion of creation of spinless particles, two cases arise. The first case is possible, when modes $k$ and cosmic time $t$ obey the inequality

$$
|k|<\left(\frac{\sigma}{12 \eta^{3}}\right)^{1 / 4}\left[5.93 \exp \left\{\left(t-t_{0}\right) \sqrt{\frac{4 \sigma}{3 \eta}}\right\}-3\right] .
$$

In this case, Bessel's function can be approximated, according to (3.15). Now, using (4.7) and (4.8) in (4.6), one can easily compute

$$
\alpha_{k}=-\frac{(3+4 i v)}{4|v|} i^{3 / 2}
$$

and

$$
\beta_{k}=\frac{3 i^{3 / 2}}{4|v|} e^{-2 i v \tau}
$$

Here, it is helpful to elaborate implications of the inequality (4.9). This inequality means that as cosmic time $\mathrm{t}$ increases beyond $t_{0}$, the approximation of Bessel's function can be valid for higher and higher modes. It implies that if at $t-t_{1}>t_{0}$, $|k|=0,1,2, \ldots\left|k_{1}\right|$, then at $t=t_{2}>t_{1},|k|=\mathrm{U}, 1, L, \ldots\left|k_{2}\right|$ wuesu $\mid n_{2} \|-\cdots_{11}$. 


\section{$K P$ Sinha and $S K$ Srivastava}

The relative probability of creating a pair of particle-antiparticle in mode $k$ isgiven by

$$
\omega_{k}=\left|\beta_{k} / \alpha_{k}\right|^{2}=\frac{9}{9+16 v^{2}} .
$$

As $\omega_{k}$, given by (4.11), is independent of $k$, so it is true for all $k$ satisfying the constraint (4.9). The absolute probability of creation of $0,1,2, \ldots$ particle-antiparticle pairs is given by

$$
\left.\left.\sum_{n=0}^{\infty}\right|_{\text {out }}\langle 0|n| 0\rangle_{\text {in }}\right|^{2}={\underset{\downarrow}{n=0}}_{k} \prod_{k} \omega_{k}^{n}\left|\alpha_{k}\right|^{1-2} .
$$

Using (4.11), one gets

$$
\sum_{n=0}^{\infty} \omega_{k}^{n}=\frac{1}{1-\omega_{k}}=\frac{9+16 v^{2}}{16 v^{2}} .
$$

From (4.10a), (4.12) and (4.13)

$$
\left.\left.\sum_{n=0}^{\infty}\right|_{\text {out }}\langle 0|\eta| 0\rangle_{\text {in }}\right|^{2}=\prod_{k} \frac{9+16 v^{2}}{16 v^{2}} \cdot \frac{16 v^{2}}{9+16 v^{2}}=1 .
$$

Equation (4.14) implies that the probability of creation of infinitely many pairs of particle-anti-particle in all modes $k$ (satisfying the constraint (4.9)) is 1 . Physically, it means that infinitely many pairs of particles will be definitely created by the rapidly expanding geometry given by the line element (2.10) with

$$
a(t)=a_{0} \sinh ^{1 / 2}\left[\frac{2}{\eta a_{0}^{2}}\left(t-t_{0}\right)+0.89\right] .
$$

The absolute probability that the vacuum remains vacuum i.e., no particle is created in any mode is given by

$$
\left.\left.\right|_{\text {out }}\langle 0 \mid 0\rangle_{\text {in }}\right|^{2}=\prod_{k}\left|\alpha_{k}\right|^{-2}=\left(\frac{16 v^{2}}{9+16 v^{2}}\right)^{1-2|k|}
$$

which is obtained using (4.10a).

The four-volume is calculated as

$$
\begin{aligned}
V_{4} & =\int_{t_{0}}^{t} a_{0}^{3} \sinh ^{3 / 2} \tau \mathrm{d} t \int \mathrm{d}^{3} x \\
& \sim 10 \cdot 72\left(\eta^{-1} \sigma^{-5}\right)^{1 / 4}\left[e^{\left(t-t_{0}\right)} \sqrt{\frac{3 \sigma}{4 \eta}}-1\right] .
\end{aligned}
$$

The decay rate of the $|0\rangle_{\text {in }}$ state per unit time per unit volume is given as

$$
\begin{aligned}
\Gamma & =-\left.\left.\frac{1}{V^{4}} \ln \right|_{\text {out }}\langle 0 \mid 0\rangle_{\text {in }}\right|^{2} \\
& =\frac{0.093 \eta^{1 / 4} \sigma^{5 / 4}(1+2|k|) \ln \left(\frac{9+4 v^{2}}{4 v^{2}}\right)}{\left[\exp \left\{\left(t-t_{0}\right) \sqrt{\left.\frac{3 \sigma}{4+\eta}\right\}}-1\right]\right.}
\end{aligned}
$$


which is obtained using the inequality (4.9) and (4.15) and (4.16). Decay of the $|0\rangle_{\text {in }}$ state means creation of particles. So decay rate of the $|0\rangle_{\text {in }}$ state per unit time per unit volume implies creation rate of particle-antiparticle pairs per unit time volume. Equation (4.17) implies that in a particular mode $k$, satisfying the condition (4.9), creation rate of particle-antiparticle pairs per unit time per unit volume increases till

$$
t=t_{1}=t_{0}+0.797 \sqrt{\eta / \sigma}
$$

attains maximum and starts decreasing when $t>t_{1}$. At time $t_{1}$, given by (4.19)

$$
|k|<8.9436\left(\sigma / 12 \eta^{3}\right)^{1 / 4}
$$

according to inequality (4.9). It shows that particle production is possible in very high modes at $t-t_{1}$. It is also clear from the inequality (4.9), that as time increases the phenomenon of particle creation can be discussed in higher and higher modes. But, as discussed above, particle creation rate $T$ gets suppressed when $t>t_{1}$ and at $t=t_{1}$ modes $k$ have upper bound given by the inequality (4.20). When $t>t_{1}$, it is possible to have $k$ such that $k \backslash>8.9436\left(\sigma / 12 e t a^{3}\right)^{1 / 4}$, but particle creation rate will be suppressed.

In the case

$$
|k| \gg\left(\sigma / 12 \eta^{3}\right)^{1 / 4}\left[5.93 \exp \left\{\left(t-t_{0}\right) \sqrt{\frac{4 \sigma}{3 \eta}}\right\}-6\right]
$$

using (4.6), (4.7), (4.8) and asymptotic expansion of $J_{l}(x)$ for large $\mathrm{x}$, as

$$
J_{l}(x) \simeq\left(\frac{\pi x}{2}\right)^{-1 / 2} \cos \left(x-\frac{\pi}{4^{1}}-\frac{\pi}{\bar{z}}, \quad\right. \text { fcl }
$$

it is found that

$$
\left|\alpha_{k}\right|^{2}=\left|\beta_{k}\right|^{2}
$$

which does not satisfy the condition (4.6c). It means that particle creation is not possible in extremely high modes.

\section{Energy of created spinless particles other than Riccions}

The components of energy-momentum of created particles can be defined as $[15,18]$

where

$$
\bar{T}^{\mu \nu}={ }_{\text {in }}\left\langle 0\left|T^{\mu \nu}\right| 0\right\rangle_{\text {in }}-{ }_{\text {out }}\left\langle 0\left|T^{\mu \nu}\right| 0\right\rangle_{\text {out }},
$$

$$
T_{\mu \nu}=\partial_{\mu} \phi^{*} \partial_{\nu} \phi-\frac{1}{2} g_{\mu v}\left[\partial^{\sigma} \phi^{*} \partial_{\sigma} \phi-\left(\xi \eta^{-1} \sigma+\lambda \sigma^{2}\right) \phi^{*} \phi\right]
$$

with

$$
\bar{T}_{n}^{0}={ }_{\text {in }}\left\langle 0\left|T_{0}^{0}\right| 0\right\rangle_{\text {in }}-{ }_{\text {out }}\left\langle 0\left|T_{0}^{0}\right| 0\right\rangle_{\text {out }}
$$

In (5.1), divergences are cancelled by taking the difference. The total energy of created particles can be calculated as

$$
E={ }_{\mathrm{j}}^{\mathrm{f}} \mathrm{d}^{3} x a^{3}(t) \bar{T}_{0}^{0}
$$


$K P$ Sinha and $S K$ Srivastava

Using (4.7)/and (4.8) in (5.1)

$$
\begin{aligned}
& { }_{\text {in }}\left\langle 0\left|T_{0}^{0}\right| 0\right\rangle_{\text {in }}=\frac{1}{2} \sum_{k}\left[\left(\left|\alpha_{k}\right|^{2}+\left|\beta_{k}\right|^{2}\right)\right]\left\{\sqrt{\frac{\sigma}{3 \eta}} a_{0}^{-3} A B V^{-1} \times\right. \\
& \frac{\left(\frac{9}{16}+v^{2}\right)}{\Gamma(1+i v) \Gamma(1-i v)} e^{-3 \tau / 2} \exp \left[i k_{a}\left(x^{a}-x^{\prime a}\right]+\right. \\
& \frac{\left(\xi \eta^{-1} \sigma+\lambda \sigma^{2}+k^{2}\right) a_{0}^{-3} A B V^{-1}}{\Gamma(1+i v) \Gamma(1-i v)} \sqrt{\frac{3 \eta}{\sigma}} e^{-3 \tau / 2} \exp \left(i k_{a}\left(x^{a}-x^{\prime a}\right)\right. \\
& +\beta_{k}^{*} \alpha_{k}^{*} a_{0}^{-3} B^{2} V^{\gamma-1} \frac{e^{-3 \tau / 2}\left(\frac{K e^{-\tau}}{2}\right)^{-2 \mathrm{iv}}}{(\Gamma(1-i v))^{2}} \\
& \left\{\sqrt{\frac{3 \eta}{\sigma}}\left(\zeta \eta^{-1} \sigma+\lambda \sigma^{2}+k^{2}\right)-\sqrt{\frac{\sigma}{3 \eta}}\left(\frac{3}{4}-i v\right)^{2}\right\} \\
& \exp \left(i k_{a}\left(x^{a}+x^{\prime a}\right)\right) \\
& +\alpha_{k} \beta_{k} a_{0}^{-3} A^{2} V^{-1} \frac{e^{-3 \tau / k} \cdot\left(\frac{K e^{-\tau}}{2}\right)^{2 i v}}{(\Gamma(1+i v))^{2}} \exp \left(-i k_{a}\left(x^{a}+x^{\prime a}\right)\right) \times \\
& \left.\left\{\sqrt{\frac{2 \eta}{\sigma}}\left(\xi \eta^{-1} \sigma+\lambda \sigma^{2}+k^{2}\right)-\sqrt{\frac{\sigma}{3 \eta}}\left(\frac{3}{4}+i \nu\right)^{2}\right\}\right]
\end{aligned}
$$

and

$$
\begin{aligned}
& { }_{\text {out }}\left\langle 0\left|T_{0}^{0}\right| 0\right\rangle_{\text {out }}=\frac{1}{2} \sum_{k} \frac{A B \exp \left(i k_{a}\left(x^{a}-x^{\prime a}\right)\right)}{\Gamma(1+i v) \Gamma(1-i v)} \\
& {\left[\sqrt{\frac{\sigma}{3 \eta}}\left(\frac{9}{16}+v^{2}\right)+\left(\xi \eta^{-1} \sigma+\lambda \sigma^{2}+k^{2}\right) \sqrt{\frac{3 \eta}{\sigma}}\right] .}
\end{aligned}
$$

Now

and

$$
\begin{aligned}
& \int \mathrm{d}^{3} x a_{0}^{3} \sinh ^{3 / 2} \tau_{\mathrm{in}}\left\langle 0\left|T_{\mathrm{o}}^{\circ}\right| 0\right\rangle_{\mathrm{in}} \\
& \simeq \frac{1}{2} \sqrt{\frac{\sigma}{3 \eta}}\left[\frac{\left(9+16 v^{2}\right)}{\left.|6| v\right|^{3}}\left(\frac{9}{8}+2 v^{2}\right)-\frac{27}{\left.|6| \mathrm{v}\right|^{3}} \sin 4 v \tau\right. \\
& \left.+\frac{3}{2} \sin 4 v \tau-\frac{9}{4|\mathrm{v}|} \cos 4 v \tau\right]
\end{aligned}
$$

$$
\begin{gathered}
\int \mathrm{d}^{3} x a_{0}^{3} \sinh ^{3 / 2} \tau_{\text {out }}\left\langle 0 \mid T_{0}^{0} \eta 0\right\rangle_{\text {out }} \\
\simeq \frac{1}{4|v|} \sqrt{\frac{I f f}{3 \eta}\left(\frac{9}{8}+2\right)}
\end{gathered}
$$




\section{Dual nature of Rica scalar}

Using (5.4) in (5.2)

$$
\begin{aligned}
E & =\frac{1}{2} \cdot \sqrt{\frac{\sigma}{3 \eta}}\left[\frac{1}{\left.|6| v\right|^{3}}\left(9+8 v^{2}\right) \times\right. \\
\left(\frac{9}{8}\right. & \left.\left.+2 v^{2}\right)-\frac{27}{\left.|6| v\right|^{3}} \sin 4 v \tau+\frac{3}{2} \sin 4 v \tau-\frac{9}{4|v|} \cos 4 v \tau\right] \\
& =3 \sigma \sqrt{\lambda} .
\end{aligned}
$$

Thus, it is found that an amount of energy, equal to $E$ will flow from the $|\rho\rangle_{\text {in }}$ to $|0\rangle$, out state produced due to rapidly expanding geometry lying between the two regions, which will contribute to the entropy of the universe.

\section{Acknowledgements}

Authors are thanlcful to Prof. S Odintsov for suggesting to use temperature dependent Coleman-Weinberg like potential for $R$-fields. One of the authors (KPS) is grateful to Council of Scientific and Industrial Research, New Delhi for financial support and the other (SKS) would like to thank the hospitality of the Centre for Theoretical Studies, Indian Institute of Science, Bangalore during the final phase of the work.

\section{References}

[1] S W Hawking and R Penrose, Proc. R. Soc. (London) A314, 529 (1970)

[2] R Utiyama and B S DeWitt, J. Math. Phys. 3, 608 (1962)

[3] K S Stelle, Phys. Rev. D16,953 (1977)

[4] E S Fradkin and A A Tseylin, Nucl. Phys. B201, 469 (1982)

[5] I G Avramidy and A O Barvinsky, Phys. Lett. B159, 269 (1985)

[6] L Parker and D J Toms, Phys. Rev. D29, 1584 (1984)

[7] I L Buchbinder, S.D Odintsov and I L Shapiro, Effective action in quantum gravity (IOP Publishing Ltd., 1992)

[8] B Whitt, Phys. Lett. B145, 176 (1984)

[9] S K Srivastava and K P Sinha, Phys. Lett. B307, 40'(1993)

[10] S K Srivastava and K P Sinha, Quantization of Ricci scalar (submitted)

[11] M B Green, J H Schwarz and E Witten, Superstring theory (Cambridge University Press, Cambridge, 1987)vol. 1

[12] J D Barrow and S Cotsakis, Phys. Lett. B214, 515 (1988); Phys. Lett. B258, 299 (1991)

[13] R D Tenreiro and M Quiros, An introduction to cosmology and particle physics (World Scientific, Singapore, 1988) p. 239

[14] S Coleman and E Weinberg, Phys. Rev. D7, 883 (1973)

[1.5] B S DeWitt, Fhys. Rep. 19, 295 (1975)

[16] L Parker, As ymptotic structure of space-time edited by F P Esposito and L Witten, (Plenum Press, New York 1977)

[17] N D Birrell and P C W Davies, Quantum fields in curved spaces (Cambridge University Press, Cambridge, 1982) p. 46

[18] E Mottola, Fhys. Rev. D31,754 (1985) 